\title{
Methicillin-Resistant Staphylococcus Aureus (MRSA) Colonisation level among Patients seen at a Tertiary Hospital in Maiduguri, Nigeria
}

\author{
${ }^{1}$ Adelowo KA, ${ }^{2}$ Okon KO, ${ }^{3}$ Denue BA, ${ }^{1}$ Ladan J, ${ }^{4}$ Tahir F, ${ }^{4}$ Uba A \\ ${ }^{1}$ Department of Immunology and Infectious Diseases, University of Maiduguri Teaching Hospital, Maiduguri \\ $\star^{2}$ Department of Medical Microbiology, Federal Medical Centre, Makurdi \\ ${ }^{3}$ Department of Medicine, University of Maiduguri Teaching Hospital, Maiduguri \\ ${ }^{4}$ Department of Biological Sciences, Abubakar Tafawa Balewa University, Bauchi.
}

${ }^{*}$ Corresponding author's E-mail: okonkenneth@gmail.com

\begin{abstract}
Methicillin-resistant Staphylococcus aureus (MRSA) is the major causative bacterial pathogen responsible for hospital and community associated infections. Active surveillance remains the key component of MRSA infection control in the hospital setting. This study examined the MRSA colonization level among patients seen at the tertiary hospital. The study was conducted at University of Maiduguri Teaching Hospital, Nigeria. A standardized questionnaire and interview was employed in enrolling 231 patients into the study and 3 anatomical body sites (nasal, axilla and groin) per patient were swabbed and analysed by standard bacteriological techniques. A total of 981 specimens were analysed, 21(2.1\%) S.aureus isolates were identified, 10(\%) MRSA and 11(\%) MSSA isolates detected. The mean age was $34.02+13.2$ years, gender distribution of $12(57.1 \%)$ male and $9(42.9 \%)$ female. Majority of S.aureus isolates were recovered from nasal compared to non-nasal origin $(<0.001)$, while no statistical significant difference was observed between the MRSA colonization and demographic variable documented. The S.aureus isolates demonstrated high resistant pattern to fusidic acid, all MRSA isolates exhibited multidrug resistant pattern and inducible phenotype. This is the first report on MRSA colonization level among patients management in a hospital setting in this geographical zone, and the S.aureus strains demonstrated high resistant to fusidic acid and exhibited high inducible phenotype which is of public health concern particularly in infection control and patient management.
\end{abstract}

Keywords: MRSA, Colonization level, Infection control, Maiduguri, Nigeria

\section{INTRODUCTION}

Staphylococcus aureus is a major bacterial pathogens responsible for hospital and community-associated infections. The anterior nares are the primary ecological niches of $S$. aureus, while other non-nasal includes throat, perineum, groin, axilla, hairline and skin. Studies have documented S.aureus colonization of both the nasal and non-nasal sites as the major predisposing risk factor in the development of staphylococcal infections in both hospital and community setting (von Eiff et al., 2000; Wertheim et al., 2005).
Of the S.aureus strain that has attracted public health attention globally has been methicillin -resistant S.aureus (MRSA). Primarily, due to its multidrug resistant pattern, high dissemination rate and difficulty in infection control within hospital setting. Over the years, the epidemiological trend of the pathogen continued to change, in its phenotypic and genotypic characteristics further compounding treatment and management of staphylococcal infections and infection control measures.

MRSA colonized individuals are highly predispose to 
developing infections, particularly those on admission in intensive care unit and patients on hemodialysis due to immume status and high antimicrobial usage (Ghasemian et al., 2010). In addition, the high degree of antibiotic consumption, particularly fluoroquniolones, macrolides, and cephalosporin could facilitate skin colonization (Graffinder and Venezia, 2002; Weber et al., 2003). In the hospital environment, colonized/ infected patients and health care remains the major reservoirs, while the hands of health care workers and clinical equipment served in furthered dissemination (Albrich and Harbarth, 2008). Active surveillance for MRSA strains have been adopted as infection control procedures in developed countries as one of the key component to understanding the epidemiological pattern, identifying the predisposing risk factors, bacteriological outcome (van Trijp et al., 2007; Robicsek et al., 2008; Matehson et al., 2008). However, adoption of such procedure in resource limited hospital in most sub-saharan African countries may be rather unfeasible because of cost implication.

In northeastern Nigeria, there is still paucity of epidemiological information on MRSA while information on colonization level among patients and healthy individual is non-existent. Therefore, this is first study to determine the prevalence of MRSA colonization level of the patients seen at the tertiary hospital in Maiduguri, Nigeria.

\section{MATERIALS AND METHODS}

This cross-sectional descriptive study was conducted at the University of Maiduguri Teaching Hospital, Maiduguri (UMTH) between January and July 2012. The hospital is a 530 bed size that provides multi-specialties medical services and training of health care professional. The study protocol was approved by UMTH institutional review board before the commencement of the study. The patients were recruited into the study based on signed informed consent and willingness to participate in the study. The study questionnaires were administered and collected information entered into the study database. Three anatomical site swabs were collected pe patients. A total of 981 swabs were collected from patients attending/or admitted to medical, surgical or general outpatient clinics.

\section{Samples collection}

The author (AK) was trained at the ENT department of UMTH, on the proper nasal swab collection procedures. For nasal swab, the labeled sterile swab was moisten in sterile normal saline, and inserted into the anterior nare, rolled several times, removed and transported to the microbiology laboratory immediately for further analysis. Similarly, aseptically cleaned area of the axilla and groin were swabbed with the moisten labeled swab. The sampled swabs were inoculated on blood and mannitol salt agar plates, incubated at $37^{\circ} \mathrm{C}$ for 24 hours. Presumptive S.aureus colonies with beta heamolysis on blood agar and yellowish colonies on mannitol salt agar plates were furthered identified by standard bacteriological procedures, colonial morphology, gram reaction, tube coagulase, catalase and DNase test (Chessborough, 2006).

Antimicrobial susceptibility testing was performed by disc diffusion method on Mueller-Hinton agar according to Clinical and Laboratory Standards Institute (CLSI) guidelines. The identified S.aureus isolates were inoculated into $5 \mathrm{ml}$ normal saline, mixed thoroughly until matching with a standard turbidity (0.5 Mac Farland). The following antimicrobial agents (manufactured by Oxoid, UK) were tested, penicillin, tetracycline, gentamicin, cotrimoxazole, erythromycin, clinidamycin, fusidic acid, ciprofloxacin, ofloxacin, oxacillin, cefoxitin, vancomycin. (OXIOD, UK). MRSA isolates were detected by an inhibition zone of $<11 \mathrm{~mm}$ to oxacillin and $<22 \mathrm{~mm}$ to cefoxitin. Multidrug resistant strain was defined as S.aureus resistant to more than 3 antimicrobial classes. The $S$. aureus isolates that indicated erythromycin resistance but susceptible to clindamycin were further analysed using the D-test described by Freibelkorn et al. (2003) in which the erythromycin and clindamycin discs were placed at $12-14 \mathrm{~mm}$ apart, and resistance classified as either inducible or constitutive. The phenotype of resistance to macrolides, lincosamides, and type B streptogramin $\left(\mathrm{MLS}_{\mathrm{B}}\right)$ was classified as constitutive $\left(\mathrm{CMLS}_{\mathrm{B}}\right)$, when the erythromycin and clindamycin discs showed resistance while inducible phenotype (iMLS $\mathrm{B}_{\mathrm{B}}$ ) was indicated by flattening of the clindamycin zone adjacent to the erythromycin discs.

\section{Statistical analysis}

The demographic variables and the frequency of MRSA occurrence was analysed using SPSS version $17^{\text {th }}$ version. Data were analysed using descriptive statistics, and statistical significant difference at $p<0.05$.

\section{RESULTS}

Of the 981 samples analysed, 21(2.1\%) S.aureus isolates were identified, $15(71.4 \%)$ recovered from nasal, $4(19.9 \%)$ and $2(9.5 \%)$ from axilla and groin respectively. The mean age of the patients was 34.02 \pm 13.2 years, gender distribution of $12(57.1 \%)$ male and $9(42.9 \%)$ female. Of the 21 S.aureus isolates, 10(47.6\%) were detected as MRSA, 9(42.9\%) from nasal and 1(4.1\%) from axilla, compared to $11(52.4 \%)$ as MSSA isolates, $6(28.6 \%)$ from nasal, $1(4.8 \%)$ axilla and $4(19.9 \%)$ groin $(<0.001)$. Demographic variables of the MRSA isolates (table 1), 4(19.9\%) were recovered from patients within the age group of 20-29years and 2(9.5\%) each in $40-$ 49 and 50-59years, 6(28.6) from female and 
240 J. Med. Med. Sci.

Table 1. Demographic variable of S.aureus isolated

\begin{tabular}{|c|c|c|c|c|c|}
\hline \multicolumn{2}{|l|}{ Demographic information } & S.aureus & MRSA & MSSA & p-value \\
\hline \multicolumn{2}{|l|}{ Mean age(years) } & \multicolumn{4}{|c|}{$34.02 \pm 13.22$} \\
\hline \multicolumn{2}{|l|}{ Nasal } & $15(71.4)$ & $9(42.9)$ & $6(28.6)$ & \\
\hline \multicolumn{2}{|l|}{ Axilla } & $2(9.5)$ & $1(4.8)$ & $1(4.8)$ & 0.001 \\
\hline \multicolumn{2}{|l|}{ Groin } & $4(19.9)$ & - & $4(19.9)$ & \\
\hline \multicolumn{6}{|l|}{ Age-group(years) } \\
\hline \multicolumn{2}{|l|}{$10-19$} & $2(9.5)$ & $1(4.8)$ & $1(4.8)$ & \\
\hline \multicolumn{2}{|l|}{$20-29$} & $8(38.1)$ & $4(19.9)$ & $4(19.9)$ & \\
\hline \multicolumn{2}{|l|}{$30-39$} & $4(19.9)$ & $1(4.8)$ & $3(14.3)$ & 0.875 \\
\hline \multicolumn{2}{|l|}{$40-49$} & $3(14.3)$ & $2(9.5)$ & $1(4.8)$ & \\
\hline \multicolumn{2}{|l|}{$50-59$} & $4(19.9)$ & $2(9.5)$ & $2(9.5)$ & \\
\hline \multicolumn{2}{|l|}{$>60$} & - & - & - & \\
\hline \multicolumn{2}{|l|}{ Sex-Male } & $12(57.1)$ & $4(19.9)$ & $8(38.1)$ & \\
\hline \multicolumn{2}{|l|}{ Female } & $9(42.9)$ & $6(28.6)$ & $3(14.3)$ & \\
\hline \multicolumn{2}{|l|}{ Source of sample-Inpatient } & $15(71.4)$ & $6(28.6)$ & $9(42.9)$ & 0.281 \\
\hline \multicolumn{2}{|l|}{ Outpatient } & $6(28.6)$ & $4(19.9))$ & $2(9.5)$ & \\
\hline \multicolumn{2}{|l|}{ Ward/Clinic-Medical } & $9(42.9)$ & $4(19.9)$ & $5(23.8)$ & \\
\hline \multicolumn{2}{|l|}{ Surgical } & $6(28.6)$ & $2(9.5)$ & $4(19.9)$ & 0.305 \\
\hline \multicolumn{2}{|l|}{ GOPD } & $6(28.6)$ & $4(19.9)$ & $2(9.5)$ & \\
\hline \multicolumn{2}{|c|}{ Previous Hospitalization (In last 6months)-Yes } & $10(47.6)$ & $6(28.6)$ & $4(19.9)$ & \\
\hline & - No & $11(52.4)$ & $4(19.9)$ & 7 (33.3) & 0.290 \\
\hline \multirow[t]{2}{*}{ Antibiotic Prescription/administration- } & Yes & $1(4.8)$ & $1(4.8)$ & $0(0.0)$ & \\
\hline & No & $20(95.2)$ & $9(43.9)$ & $11(1.1)$ & 0.214 \\
\hline \multirow[t]{2}{*}{ Indwelling Medical devices- } & Yes & $6(28.6)$ & $3(14.3)$ & $3(52.4)$ & \\
\hline & No & $15(71.4)$ & 7 (33.3) & $8(38.1)$ & 0.893 \\
\hline \multirow[t]{2}{*}{ Medical/Surgical Procedures } & Yes & $4(19.9)$ & $2(9.5)$ & $2(9.5)$ & \\
\hline & No & $17(81.0)$ & $8(38.1)$ & $9(43.9)$ & 0.918 \\
\hline \multirow[t]{2}{*}{ Associated clinical conditions } & Yes & $6(28.6)$ & $2(9.5)$ & $4(19.9)$ & \\
\hline & No & $15(71.4)$ & $8(38.1)$ & 7 (33.3) & 0.480 \\
\hline
\end{tabular}

Table 2. Resistance phenotype of S.aureus strains

\begin{tabular}{lcc}
\hline Resistance profile & MRSA & MSSA \\
\hline Ofx & & 1 \\
Tet & \multicolumn{1}{c}{1} \\
Fa & 1 & 1 \\
Tet + Pen + Ery & & 1 \\
Tet + Sxt + Fa & & 1 \\
Pen + Ofx + Fa & 1 & \\
Tet + Pen + Ery + Ofx & 3 & \\
Pen + Ery + Gen +Sxt + Fa & 2 & \\
Pen + Ery + Tet +Sxt +Fa & 1 & \\
Pen +Ery + Tet +Sxt +Ofx & & 1 \\
Pen +Ofx +Cip +Sxt + Fa & 1 & \\
Pen + Ery + Tet+ Sxt+ Gen & & 1 \\
Pen + Cd, + Ery + Cip + Sxt + Fa & & 1 \\
Pen + Cip + Gen+ Ery+ Sxt+Fa & 1 & \\
Pen + Cd + Gen + Tet + Sxt+Fa & & \\
\hline
\end{tabular}

Ofx-ofloxacin, Tet-tetracycline, Fa-fusidic acid, Pen-penicillin, Sxt-cotrimoxazole, Gen-gentamicin, Cip-ciprofloxin,Ery-erythromycin, Cd-Clindamycin. 


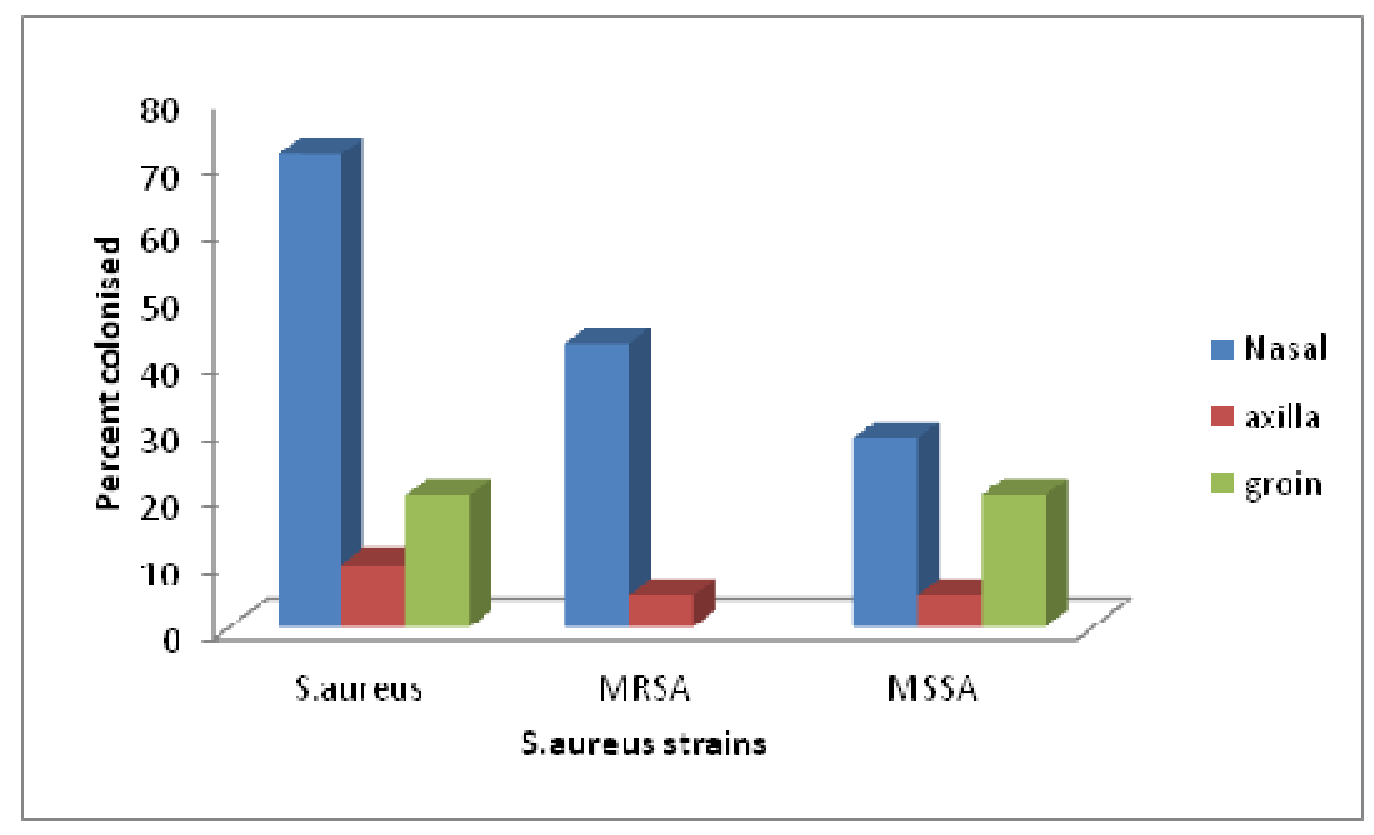

Figure 1. Prevalence of S.aureus (MRSA, MSSA) strains colonization level

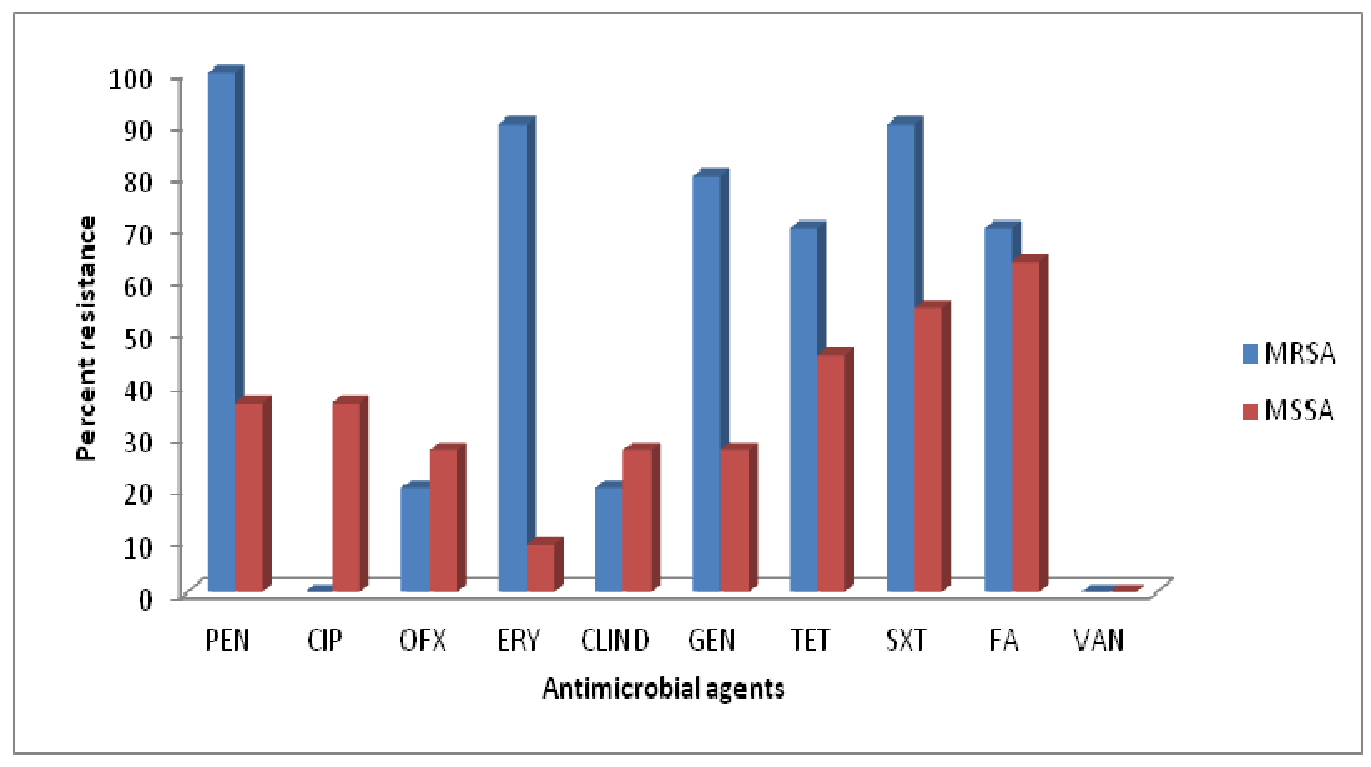

Figure 2. Antimicrobial resistance pattern of S.aureus strains

$4(19.9 \%)$ males, $6(28.6 \%)$ inpatients and $4(19.9 \%)$ outpatient, $4(19.9 \%)$ each on admission in the medical ward and patients seen at the GOPD and $2(9.5 \%)$ in the surgical wards.

There was no statistical significant difference observed between MRSA colonization and the predisposing risk factors documented in the questionnaires, 9(43.9\%)MRSA colonized patients had no antibiotic prescriptions /administration, $7(33.3 \%)$ with no indwelling medical devices, $8(38.1 \%)$ had no medical or surgical and $8(38.1 \%)$ no associated clinical conditions respectively, except $6(28.6 \%)$ had medical history of previous hospitalization.

The antimicrobial resistance pattern of the S.aureus isolates as presented in figure 1, MRSA isolates showed high resistance to penicillin(100\%), tetracycline(70\%), cotrimoxazole( $90 \%)$, gentamicin $(80 \%)$, erythromycin $(90 \%)$ and fusidic acid(70\%). While high sensitivity to ciprofloxacin(97\%), of loxacin( $80 \%$ ) and clindamycin(80\%). The MSSA isolates showed high 
resistance to fusidic acid (60\%), cotrimoxazole(52\%), tetracycline $(50 \%)$ and low resistance to pencillin and ciprofloxacin(39\%), ofloxacin(30\%), erythromycin(10\%) and clindamycin and gentamycin(25\%). Statistical significance difference was observed in the resistance pattern of MRSA and MSSA isolates to penicillin and clindamycin $(<0.001)$. All the 10 MRSA isolates exhibited multidrug resistant (MDR) pattern and inducible phenotype, while only $5 \mathrm{MSSA}$ isolates demonstrated MDR pattern

\section{DISCUSSION}

As MRSA remain the major causative agent of hospital and community associated infections, with attendant clinical and societal consequence. Epidemiological understanding of MRSA predisposing risk factors serves as the template for appropriate antimicrobial treatment/management of staphylococcal infections and infection control measures. This information can only be obtained from active surveillance of MRSA in hospital setting. Therefore, this findings had revealed salient epidemiological information on MRSA

In this study, the overall S.aureus colonisation level was $21(2.1 \%)$, in which 10(47.6\%) MRSA and $11(52.4 \%)$ MSSA strains were detected, with majority of the strains recovered from nasal origin. The S.aureus colonisation level of $2.1 \%$ is low, when compared to the reported prevalence level of nasal carriage of S.aureus ranged between 16.8\%-90\% (Alghaithy et al., 2000; Goyal et al., 2002; Askarian et al., 2009; Heininjar et al., 2007). The S.aureus colonisation varies with geographical location, studied population, type of hospital and sampling procedures (Bell and Turndge, 2002). The MRSA colonisation level is considered to be low, when compared with other studies which ranged between 5.9\%-15.6\% (Nahimana et al., 2006; Wang et al., 2009). Lower MRSA colonization have been reported in some studies, $7.7 \%$ in Serbia (Cirkovic et al., 2014), 9.7\% in Ukarine (Netsvyetayeva et al., 2014), while high level reported in other studies, $21.2 \%$ in Sao Tome and Principle (Conceicao et al., 2013), 36.8\% in Iran (Mohajari et al., 2013), 66.4\% in Scotland (Matheson et al., 2012), 50\% in Libya (El-Bouri and El-Bouri, 2013), $50.5 \%$ in Ireland (Huggins et al., 2011). Observed differences in MRSA colonisation level between studies countries and hospitals may be best explained by various factors, microbiological methods (from sampling technique to culture media), local infection control standards, and the local prevalence of MRSA.

As reported in some studies, MRSA colonisation level is known to be influenced by age-and gender dependent and hospital facilities (Askarian et al., 2009; Heininger et al., 2007; Ghasemian et al., 2010; Parvez et al., 2010; Huh et al., 2012). In this study, we observed no statistical significant difference between the demographic variables, such as age, gender and antimicrobial usage and MRSA colonisation level. Nevertheless, relatively few MRSA strain detected were too small to deduce the effect of these demographic variables on the MRSA colonisation level as they might not serve as good epidemiological representation of colonisation level.

In Europe and US, routine screening of patients particularly nasal before, during and discharge has formed the part of routine infection control and intervention measures of MRSA infection (Matheson et al., 2008). But some studies found out that using only nasal alone in detection of MRSA colonisation level shows low specificity and sensitivity, compared with the rate from combination of nasal with other body site like axilla, groin and throat (Huggins et al., 2011; El-Bouri and El-Bouri, 2013). Even though, evaluation of anatomical site was not part of the objective of this study, nevertheless, statistical significant difference was observed between the S.aureus identified and the anatomical body sites sampled.

Studies have shown relationship between methicillin resistance and resistance to other antibiotics (Nahimana et al., 2006, Chen et al., 2011). In sub-saharan African countries, high resistance to commonly prescribed and administered agent is a common norm, because these agents can easily be purchased over the counter, administered for variety of clinical condition and some are substandard. Noteworthy, is the high fusidic acid resistant pattern observed in this study, as this topical agent is neither used routinely in our hospital for decolonization nor decontamination of colonized/or infected patients, and the reason for the patternis unclear. Similar pattern was exhibited by S.aureus isolates recovered from anterior nares of ruminant animals slaughtered for human consumption and contact person in Maiduguri (Maisayama et al., 2014). This pattern may be assumed to be an evolving epidemiological trend that required further research studies focusing on molecular characterization of the fusidic acid resistance gene. Other studies conducted in Africa have reported high fusidic acid resistant pattern in S.aureus isolated in studies in Morocco, Ivory Coast, Senegal and Cameroon (Belabbes et al., 2001; Kesah et al., 2003; Elhamzaoui et al., 2009). Apart from the fusidic acid resistant MRSA strain, the strains also demonstrated multidrug resistant pattern and inducible phenotype which is of public health implication because of is possible dissemination within hospital thus making infection control approach difficult. In addition, this pattern has negative implication on the treatment and management of staphylococcal infections due to possible chemotherapeutic failure.

From the epidemiological perspective, the finding of this study had provided salient information on MRSA colonisation level in a geographical zone with paucity of data. However, there are limitations in the study,(i) the number of S.aureus isolates were few, (ii) Data collection 
was based on interview and patient record- which subject to error, and poor documentation, (iii) the duration of study was relatively short and high risk unit like ICU and renal unit were not included.

In conclusion, the findings had shed epidemiological light on MRSA colonisation level among patient on admission and those seen on outpatient basis. The high resistant pattern observed with fusidic acid is of public health concern particularly within the hospital setting, and the need for further studies on the molecular characterization of fusidic acid resistance gene.

\section{ACKNOWLEDGEMENT}

We acknowledged the staff of department of Medical Microbiology, University of Maiduguri Teaching Hospital, Maiduguri and Department of Biological Sciences, Abubakar Tafawa Balewa University, Bauchi for their support in the analysis of the isolates.

\section{REFERENCES}

Albrich WC, Harbarth (2008). Health care worker source, vector of MRSA. Lancet Infect. Dis.8;289-301.

Alghaithy AA, Bilal NE, Gedebou M, Weily AH (2000). Nasal carriage and antibiotic resistance of Staphylococcus aureus isolates from hospital and non-hospital personnel in Abba, Saudi Arabia. T. Roy. Trop. Med Hy.94, 504-607

Askarian M, Zeinalzadeh A, Japori A, Alborzi A, MemishZA (2009). Prevalence of nasal carriage of methicillin resistant Staphylococcus aureus and its antibiotic susceptibility pattern in health care worker at Nameji hospital Shuraz, Iran. Int. J. Infect. Dis. 13(50, 244-247.

Belabbes H, Elmadeghari W, Hanshimi K, Marih H, Zarouali K, Benbachir M (2010). Antibiotic resistance of Staphylococcus Aureus isolated from community and nosocomial infections in Casabalanca Morocco. Med. Mal. Infect. 39(1) 25-28.

Bell JM, Turnidge JD (2002). High prevalence of oxacillin-resistant Staphylococcus aureusisolates from hospitalized patients in AsiaPacific and South Africa: results from SENTRY Antimicrobial Surveillance Program, 1998-1999. Antimicrob. Agents Chemother. 46:879-881.

Cheesborough M (2006). District Laboratory Practice in Tropical Countries, Microbiologysecond edition Cambridge University Press.2:158-195.

Chen CB, Chang HC, Huang YC (2010). Nasal methicillin-resistant Staphylococcus aureus carriage among intensive care unit hospitalized adult patients in a Taiwanese medical centre; one point -point prevalence, molecular charateritic and risk factor for carriage. J. Hosp. Infect.74,238-244.

Cirkovic I, Dukic S, Carevic B, Mazic N, Mioljevic V, Stepanovic S (2014). Methicllin resistant Staphylococcus aureus nasal carriage among hospitalized patients and health care workers in medical centre of Serbia. Arch. Biol. Sci. Belgrade, 66(1)87-92

CSLI (2006). Performance Standards for Antimicrobial Disk Susceptibility Tests; Sixteenth International Supplement Clinical and Laboratory Standards Institute, Wayne, PA, USA. Document 11100S16

El-Bouri K, El-Bouri W (2013). Screening cultures for detection of methicillin-resistant Staphylococcus aureus in a population at high risk for MRSA colonisation: identification of optimal combinations of anatomical sites. Libyan J. Med., 8: 22755

Elhamzaoni S, Benonda A, Allal F, Abougal R, Elouannass R (2009). Antibiotic susceptibility of Staphylococcus Aureus strain isolated in the University hospital in Rabat, Morocco. Med. Mal. Infect, 39(1) 891-5
Fiebelkorn KR, Crawdford SA, McElmeal MI, Jorgensen JH (2003). Practical disk diffusion method for detection of inducible clindamycin resistance in Staphylococcus aureus and coagulasenegative staphylococci. J. Clin. Microbiol., 41: 4740-4744.

Ghaseman R, Najafi N, Makhlough A, Khademloo A (2010). Frequency of nasal carriage of Staphylococcus aureus and their antimicrobial resistant pattern of patients on heamodialysis. Iran. J. Kidney Dis. 4(3),218-22.

Goyal R, Das S, Alathur M (2002). Colonisation of methicillin resistant Staphylococcus aureus among health care workers in a tertiary care hospital of Dehli. Ind. J. Med. Sc.56, 321-324.

Heininger U, Datta F, Gervaix A, Schaad UB, Berger C, Vandaux B, Hitzler C, Kind M, Gnehm C, Hamspeter E, Reno F, and the Pigs/MRSA study group. (2007). Prevalence of nasal colonization with methiclllin resistant Staphylococcus aureus in children a multicenter cross sectional study. Peadiatric. Infect. Dis. J. 26(6) 544-546

Higgins A, Gethin G, Lynch M (2011). Prevalence of MRSA colonization among at risk patients at admission to an Irish hospital:2007 to 2009 inclusive. Int. J. Infect. Control., v7:i1

Huh HJ, Kim ES, Chae SL (2012). Methicillin resistant Staphylococcus aureus in nasal surveillance swab at a intensive care unit; an evaluation of the lightcycler MRSA advanced test. Ann. Lab. Med. 32(6), 407-12

KesahC, Ben Redjeh S, Odugbemi TO, Boye CSB, DossoM,Ndinye JO, Achila S, Koulla-Chirro et al(2003).Prevalence of methicillinresistant Staphylococcus S

Mai-siyama IB, Okon KO, Adamu NB, Askira UM, IsyakaTM,Adamu SG, Mohammed $\quad \mathrm{A}(2014)$. Methillin-resistant Staphylococcus Aureus(MRSA) colonization level among ruminanat animals slaughtered for human consumption and contact persons in Maiduguri, Nigeria.AJMR,8(27),2643-2649.

Matheson A, Christie P, Stari T, Kavanagh K, Gould IM, Masterton R (2012) Nasal swab screening for methicillinresistantStaphylococcus aureus*how well does it perform?A cross-sectional study. Infect. Control Hosp. Epidemiol. 2012;33: 803_8.

McGowan JEjr, Tenover FC (2004). Confronting bacterial resistance in health care setting; a crucial role for microbiologist. Nat. Rev. Microbiol. 2(3) 251-8

Mohajeri P, Izadi B, Rezaei M, Farahami A (2013). Frequency distribution of hospital acquired MRSA nasal carriage among hospitalized patients in west of Iran. Jundishapur J. Microbiol. 6(9) 9076

Moniri R, Musrv GA, Fadau W (2009). The prevalence of methicillinresistant Staphylococcus aureus in hospitalized patients. Pak. J. Med. Sci. 25(4),656-659

Nahimana I, Francioli P, Blanc D (2006). Evaluation of three chromogenic media(MRSA ID, MRSA Select and CHROMO agar MRSA) and ORSA for surveillance of methicillin-resistant Staphylococcus aureus. Clin. Microbiol. Infect. 12,1168-1179.

Netsvyetayeva I, Fraczek M, Piskorska K, GolasM, Sikora M, Mlynarczyk A, Swoboda-Kopec E, Marusza W, Palmieri B, lannitti $\mathrm{T}$ (2014). Staphylococcus aureus nasal carriage in Ukraine: antibacterial resistance and virulence factorencoding genes.BMC Infectious Diseases 14:128

Parvez N, Jinadatra C, Fader R, Huber TN, Robertson, Kjar D (2010). Universal MRSA nasal surveillance; characterization of outcome at a tertiary care centre and implication for infection control. South Med. J. 2010, 103(11) 1084-91

Robicsek A, Beaumont JL, Paule SM, Hacek DM, Thomson RB, Jr. Kaul KL, King P, Peterson LR (2008). Universal surveillance for methicillin-resistant Staphylococcus aureusin 3 affiliated hospitals. Ann. Intern. Med. 148:409-418.

Saxena AK, Pauhotra BR (2003). The prevalence of nasal carriage of Staphylococcus aureus and associated vascular access related septicemia among patients on heamodialysis in Al-Hasa region of Saudi Arabia. J. Kidney Dis. Transpl., 14 30-8.

Scarnato F, Mallaret MR, Croize J, Koubenan DR, Dubois M, Maitre A (2003). Incidence and prevalence of methicillin resistant Staphylococcus aureus among health care worker in geriatric department; relevance to preventive measures. Infect. Control Hosp. Epidemiol. 24(6), 456-458. 
Shakya B, Shoestha S, Mitra Y (2010). Nasal carriage rate of methicillin resistant Staphylococcus aureus among patients at National Medical College Teaching Hospital, Birging Nepal Med. Coll. J. 2010, 12(1), 26-9

Theaker C, Ormond-Walshe S, Azadian B, Jani N (2001). MRSA in the critically ill. J. Hospital Infection,48; 98-102.

vanTrijp MJ, Melles DC, Hendriks WD, Parlevliet GA, Gommans M, Ott A (2007). Successful control of widespread methicillin-resistant Staphylococcus aureuscolonization and infection in a large teaching hospital in the Netherlands. Infect. Control Hosp. Epidemiol. 28:970-975.

vonEiff C, Becker K, Machka K, Stammer H, Peters G (2001). Nasal carriage as a source of Staphylococcus aureusbacteremia. Study Group. N. Engl. J. Med. 344; 11-16.
Wang CY, Wu VC, Chen YM, Su CT, WuKD, Hsueh PR (2010). Nasal carriage of methicillin resistant Staphylococcus aureus among patients with end-stage renal disease. Infect Control Hosp. Epidemiol. 2010 30,93-94

Weber SC, Gold HS, Hooper DC (2003). Fluoroquinolones and risk of methicillin-resistant Staphylococcus aureus in hospitalized patients. Emerging Infectious Diseases, 9; 1415-22

Werthein HFL, Melles DC, Vos MC, van Leeuwen W, van Belkum A, Verbury HA, Nouwen JL (2005). The role of nasal carriage in Staphylococcus aureus infection. Lancet. Infect. Dis. 5(12) 751 62, 364: 703-704

How to cite this article: Adelowo KA, Okon KO, Denue BA, Ladan J, Tahir F, Uba A (2014). Methicillin-Resistant Staphylococcus Aureus (MRSA) among Patients seen at a Tertiary Hospital in Maiduguri, Nigeria. J. Med. Med. Sci. 5(10):238-244 